Table. 1. Activity Disease Index in PsA

\begin{tabular}{lc}
\hline ACTIVITY INDEX & $\mathbf{n = 4 2}$ \\
\hline DAPSA & $14.45(9.72-23.92)$ \\
DAPSA & 3 \\
$\leq 4$ REMISSION & 16 \\
$>4$ y 14 low disease activity & 17 \\
$>14$ y 28 moderate disease activity & 3 \\
$>28$ high disease activity & $14.00(8.00-23.00) / 41^{*}$ \\
cDAPSA & $9(25) / 36$ \\
MDA & $2.20(0.20-6.80) / 41^{*}$ \\
PASI & \\
& \\
*Expressed in median and interquartiles.Qualitative variables expressed in frequency and &
\end{tabular}

Table. 2. Lipid Profile in PsA patients.

\begin{tabular}{lc}
\hline Cholesterol (mg/dl) & $194.5(164.8-218.2)$ \\
HDL (mg/dl) & $48.00(37.00-57.00)$ \\
LDL (mg/dl) & $114.5(78.5-140.8)$ \\
TG (mg/dl) & $139.50(89.25-191.20)$ \\
\hline
\end{tabular}

Expressed in median and interquartiles.

Disclosure of Interests: None declared

DOI: 10.1136/annrheumdis-2020-eular.2185

\section{AB0831 COMPARISON OF DIFFERENT REMISSION INDICES IN PATIENTS WITH PSORIATIC ARTHRITIS: A POST HOC ANALYSIS OF DATA FROM PHASE 3 TOFACITINIB STUDIES}

E. Schneeberger ${ }^{1}$, G. Citera ${ }^{1}$, P. Nash ${ }^{2}$, J. S. Smolen ${ }^{3}$, P. J. Mease ${ }^{4}$ E. Soriano ${ }^{5}$, C. Helling ${ }^{6}$, A. E. Szumski ${ }^{7}$, R. Mundayat ${ }^{8}$, D. Graham ${ }^{9}$, D. Ponce de Leon ${ }^{10} .{ }^{1}$ Instituto de Rehabilitación Psicofísica, Buenos Aires, Argentina; ${ }^{2}$ Griffith University, Brisbane, Australia; ${ }^{3}$ Medical University of Vienna, Vienna, Austria; ${ }^{4}$ Swedish Medical Center and University of Washington, Seattle, United States of America; ${ }^{5}$ Hospital Italiano de Buenos Aires, Buenos Aires, Argentina; ${ }^{6}$ Pfizer Inc, Buenos Aires, Argentina; ${ }^{7}$ Pfizer Inc, Collegeville, United States of America; ${ }^{8}$ Pfizer Inc, New York, United States of America; ${ }^{9}$ Pfizer Inc, Groton, United States of America; ${ }^{10}$ Pfizer Inc, Lima, Peru

Background: An international task force has agreed that remission and low disease activity (LDA) are treatment targets for patients (pts) with PsA, and recommends the Disease Activity Index in Psoriatic Arthritis (DAPSA) and minimal disease activity (MDA) to assess disease activity states. ${ }^{1}$ Tofacitinib is an oral Janus kinase inhibitor for the treatment of PsA.

Objectives: In this post hoc analysis, we compared DAPSA LDA with MDA, and DAPSA remission with very low disease activity (VLDA) and DAS28-3(CRP) remission, in pts with PsA receiving tofacitinib.

Methods: Data were pooled from 2 Phase 3 studies (OPAL Broaden [12 months; NCT01877668]; OPAL Beyond [6 months; NCT01882439]) for pts receiving tofacitinib 5 or $10 \mathrm{mg}$ twice daily (BID) or placebo (PBO). DAPSA was determined by summing: swollen joint count (SJC66); tender/painful joint count (TJC68); Patient's Global Assessment of Arthritis (PtGA; visual analogue scale [VAS]); pain (VAS); and CRP. Pts were classified as achieving MDA or VLDA when meeting $\geq 5$ (MDA) or 7 (VLDA) of the following criteria: TJC68 $\leq 1$; SJC6 $\leq 1$; Psoriasis Activity and Severity Index $\leq 1$ or body surface area $\leq 3 \%$; pain (VAS) $\leq 15$; PtGA (VAS) $\leq 20 ; \mathrm{HAQ}-\mathrm{DI} \leq 0.5$; tender entheseal points (using Leeds Enthesitis Index [LEI]) $\leq 1$. A logistic regression model was used to assess demographic and baseline characteristics as predictors of a trend in DAPSA scores at Month (M)3. DAPSA LDA ( $\leq 14)$, MDA, DAPSA remission (DAPSA $\leq 4$ ), VLDA and DAS28-3(CRP) remission (DAS28-3[CRP]<2.6) rates were compared at $\mathrm{M} 1, \mathrm{M} 3$ and $\mathrm{M} 6$ for pts receiving tofacitinib $5 \mathrm{mg}$ BID and at M6 for pts receiving tofacitinib 5 or $10 \mathrm{mg} \mathrm{BID.} \mathrm{Agreement} \mathrm{between}$ disease activity indices at M6 was evaluated using a kappa test. The percentage of tofacitinib-treated pts who achieved MDA, VLDA and non-response was reported at M6, stratified by achievement of DAPSA LDA, remission or non-response.

Results: This analysis included 709 pts: tofacitinib $5 \mathrm{mg} \mathrm{BID,} n=237$; tofacitinib $10 \mathrm{mg} \mathrm{BID}, \mathrm{n}=236 ; \mathrm{PBO}, \mathrm{n}=236$. At $\mathrm{M} 3$, older patients treated with tofacitinib, and tofacitinib- or PBO-treated pts with higher baseline SJC66, TJC68, PtGA VAS, HAQ-DI, LEI and Pain VAS, were significantly $(p<0.05)$ more likely to have higher DAPSA. DAPSA LDA, MDA, remission (DAPSA and DAS28-3[CRP]) and VLDA rates generally increased from $M 1$ to $M 6$ for patients receiving tofacitinib $5 \mathrm{mg}$ BID (Figure a). At M6, most tofacitinib-treated pts who achieved MDA, and all who achieved VLDA, were also in DAPSA remission or LDA (Figure b). At least moderate agreement (defined by kappa values 0.41-0.60) was observed between DAPSA LDA and MDA, and between DAPSA remission and VLDA, with both doses of tofacitinib at M6 (Figure c).

Conclusion: Remission and LDA rates generally increased over time in pts with PsA receiving tofacitinib. DAPSA LDA showed moderate agreement with MDA, and DAPSA remission showed at least moderate agreement with VLDA, confirming that DAPSA and MDA are useful measurement tools to assess disease activity in pts with PsA treated with tofacitinib.

References:

[1] Smolen et al. Ann Rheum Dis 2018;77:3-17.

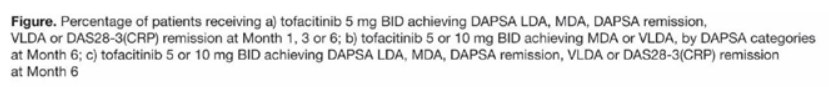
at $M$

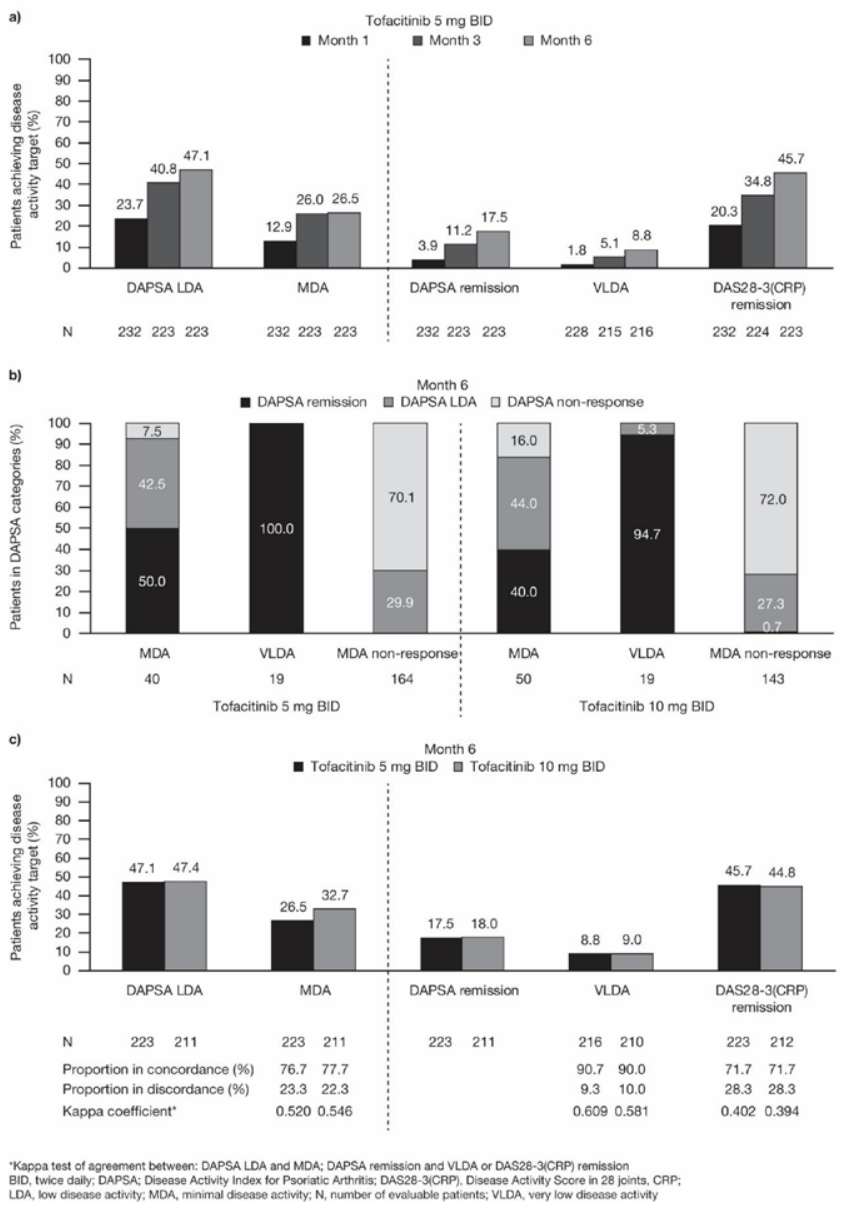

Acknowledgments: Study sponsored by Pfizer Inc. Medical writing support was provided by Sarah Piggott of CMC Connect, McCann Health Medical Communications, and funded by Pfizer Inc.

Disclosure of Interests: Emilce Schneeberger: None declared, Gustavo Citera Grant/research support from: AbbVie, Amgen, Eli Lilly, Gema, Genzyme, Novartis and Pfizer Inc, Consultant of: AbbVie, Amgen, Eli Lilly, Gema, Genzyme, Novartis and Pfizer Inc, Peter Nash Grant/research support from: AbbVie, Bristol-Myers Squibb, Celgene, Eli Lilly and Company, Gilead, Janssen, MSD, Novartis, Pfizer Inc, Roche, Sanofi, UCB, Consultant of: AbbVie, Bristol-Myers Squibb, Celgene, Eli Lilly, Gilead, Janssen, MSD, Novartis, Pfizer Inc, Roche, Sanofi, UCB, Speakers bureau: AbbVie, Bristol-Myers Squibb, Celgene, Eli Lilly, Gilead, Janssen, MSD, Novartis, Pfizer Inc, Roche, Sanofi, UCB, Josef S. Smolen Grant/research support from: AbbVie, AstraZeneca, Celgene, Celltrion, Chugai, Eli Lilly, Gilead, ILTOO, Janssen, Novartis-Sandoz, Pfizer Inc, Samsung, Sanofi, Consultant of: AbbVie, AstraZeneca, Celgene, Celltrion, Chugai, Eli Lilly, Gilead, ILTOO, Janssen, Novartis-Sandoz, Pfizer Inc, Samsung, Sanofi, Philip J Mease Grant/ research support from: Abbott, Amgen, Biogen Idec, BMS, Celgene Corporation, Eli Lilly, Novartis, Pfizer, Sun Pharmaceutical, UCB - grant/research support, Consultant of: Abbott, Amgen, Biogen Idec, BMS, Celgene Corporation, Eli Lilly, Novartis, Pfizer, Sun Pharmaceutical, UCB - consultant, Speakers bureau: Abbott, Amgen, Biogen Idec, BMS, Eli Lilly, Genentech, Janssen, Pfizer, UCB speakers bureau, Enrique Soriano Grant/research support from: AbbVie, Eli Lilly, GlaxoSmithKline, Novartis, Pfizer Inc, Sandoz, Consultant of: AbbVie, Eli Lilly, 
GlaxoSmithKline, Novartis, Pfizer Inc, Sandoz, Speakers bureau: AbbVie, Amber, Bristol-Myers Squibb, Eli Lilly, Novartis, Pfizer Inc, Roche, Claudia Helling Shareholder of: Pfizer Inc, Employee of: Pfizer Inc, Annette E Szumski Shareholder of: Pfizer Inc, Employee of: Pfizer Inc, Rajiv Mundayat Shareholder of: Pfizer Inc, Employee of: Pfizer Inc, Daniela Graham Shareholder of: Pfizer Inc, Employee of: Pfizer Inc, Dario Ponce de Leon Shareholder of: Pfizer Inc, Employee of: Pfizer Inc DOI: 10.1136/annrheumdis-2020-eular.948

\section{AB0832 IDENTIFICATION OF NATIVE AND CITRULLINATED AUTOANTIBODIES TO PSORIASIS RELATED-ANTIGEN PSOP27 IN SYNOVIAL FLUIDS OF PATIENTS WITH PSORIATIC ARTHRITIS}

M. Slobodkin ${ }^{1,2}$, S. Gertel ${ }^{2,3}$, A. Polachek ${ }^{2,3}$, V. Furer ${ }^{2,3}$, O. Elkayam ${ }^{2,3} \cdot{ }^{1}$ Tel Aviv Sourasky Medical Center, Internal medicine D, Tel-Aviv, Israel; ${ }^{2}$ Sackler Faculty of Medicine, Tel-Aviv University, Tel-Aviv, Israel; ${ }^{3}$ Tel Aviv Sourasky Medical Center, Department of Rheumatology, Tel-Aviv, Israel

Background: Psoriatic arthritis (PsA) is an inflammatory joint disease. Unlike rheumatoid arthritis (RA), PsA is a "seronegative disease", with no diagnostic biomarkers, hence diagnosis is based on clinical evaluation alone. PsA is closely associated with psoriasis ( $\mathrm{PsO}$ ), with up to $1 / 3$ of psoriatic patients developing PsA via unknown mechanism

PsoP27 is an antigen present in mast cells in psoriatic lesions and absent in skin uninvolved by psoriasis or in healthy controls (1), thus playing a significant role in inflammatory reaction in the psoriatic skin lesion (2). Its levels in skin lesions correlate with psoriasis activity (3). Though speculated to be involved in other inflammatory processes, this antigen has not been investigated in relation to PsA.

Objectives: Our aim was to identify and determine the level of native and citrullinated PsoP27 antibodies (Ab) in serum and synovial fluid of patients with PsA compared to RA and osteoarthritis (OA), exploring a potential common inflammatory pathway in $\mathrm{PsA}$ and $\mathrm{PsO}$.

Methods: Synovial fluid (SF) and serum of PsA ( $n=35$, m:f 24:11, median age 48, PsA median duration $8 Y$, PsO median duration 15Y) and RA ( $n=11$, m:f 2:9, median age 60, median RA duration 13.5Y) patients were analyzed for the level of native and citrullinated PsoP27 Ab. SF derived from OA ( $n=13$, m:f $1: 12$, median age 77$)$ patients and sera of healthy donors $(n=31)$ were used as controls. Samples were analyzed by ELISA

Results: SF levels of native and citrullinated PsoP27 Ab were significantly higher in PsA and RA compared to OA patients (Table 1).

Table 1. PsoP27 level (Optical density, OD) in SF of patients with psoriatic arthritis (PsA), rheumatoid arthritis (RA), and osteoarthritis (OA).

\begin{tabular}{lccc}
\hline PsoP27 -SF & PsA & RA & OA \\
& 0.640 & 0.855 & 0.283 \\
median & 0.298 & 0.286 & 0.28 \\
SD & 0.0082 & 0.0023 & - \\
P value (comp. to OA) & 0.0637 & - & 0.0023 \\
P value (comp. to RA) & & & \\
Cit-PsoP27- SF & 0.766 & 0.982 & 0.378 \\
median & 0.373 & 0.348 & 0.279 \\
SD & 0.003 & 0.001 & - \\
P value (comp. to OA) & 0.14 & - & 0.001 \\
P value (comp. to RA) & & & \\
\hline
\end{tabular}

Significant correlation was observed between the SF levels of both forms of PsoP27 Ab and the swollen joints count (Native: $p=0.029, r=0.39$, Cit: $p=0.041$, $r=0.369$ ), psoriasis area and severity index (PASI) score (Native: $p=0.011, r=0.56$, Cit: $p=0.008, r=0.369$ ), and CRP levels (Native: $p=0.017, r=0.446$, Cit: $p=0.03$, $r=0.408)$. In contrast, in RA patients there was no correlation between SF levels of PsoP27 Ab and the swollen joint count or CRP levels.

Both forms of PsoP27 Ab were detected in sera of all study groups, at a similar level (Table 2).

Table 2. PsoP27 level (OD) in sera of patients with psoriatic arthritis (PsA), rheumatoid arthritis (RA), and healthy controls (HC).

\section{PsoP27- Serum}

\section{median}

SD

$P$ value (comp. to $\mathrm{HC}$ )

$P$ value (comp. to RA)

Cit-PsoP27- Serum

median

SD

$\mathrm{P}$ value (comp. to $\mathrm{HC}$ )

$P$ value (comp. to RA)

$\begin{array}{ccc}\text { PsA } & \text { RA } & \text { HC } \\ 0.184 & 0.235 & 0.219 \\ 0.137 & 0.097 & 0.141 \\ 0.044 & 0.746 & - \\ 0.055 & - & 0.746 \\ & & \\ 0.317 & 0.232 & 0.321 \\ 0.298 & 0.143 & 0.160 \\ 0.767 & 0.076 & - \\ 0.044 & - & 0.076\end{array}$

Conclusion: We determined for the first time the presence of antibodies to psoriatic-related autoantigen PsoP27, in SF of PsA, RA and OA patients. Low SF level of PsoP27 Ab in OA compared to a high Ab level in RA and PsA may suggest a potential new biomarker discriminating between inflammatory arthritis versus OA. Furthermore, we showed a positive correlation between the SF levels of antibodies to PsoP27 in SF and disease activity in PsA, but not in RA. Also, we demonstrated the presence of citrullination and antibodies against citrullinated peptides in PsA, a process thought to be specific to RA. Our results suggest that antibodies to PsoP27 in SF may be a potential biomarker in PsA, both for diagnosis and disease assessment.

References:

[1] Iversen OJ, Lysvand H, Jacobsen T, Bergh K, Lie BA. Arch Dermatol Res. 1995;287(5):503-5

[2] Iversen OJ, Lysvand H, Slupphaug G. Clin Immunol. 2017;174:10-7.

[3] Dalaker M, Jacobsen T, Lysvand H, Iversen OJ. Acta Derm Venereol. $1999 ; 79(4): 281-4$

Disclosure of Interests: None declared

DOI: 10.1136/annrheumdis-2020-eular.3698

\section{AB0833 $\quad$ COMORBIDITY IN PSORIATIC ARTHRITIS WITH AND WITHOUT ENTHESITIS}

D. Somov ${ }^{1}$, M. Muradiants ${ }^{1}$, K. Kotkovskaia ${ }^{1}$, A. Kotlyarova ${ }^{1}$, M. Mariia ${ }^{1}$,

A. Pogarskaya ${ }^{1}$, N. Shostak ${ }^{1}$, A. Muradyants ${ }^{1}$, A. Klimenko ${ }^{1}$, N. Babadaeva ${ }^{1}$

${ }^{1}$ Pirogov Russian National Research Medical University, Moscow, Russian

Federation

Background: Psoriatic arthritis (PsA) is a chronic systemic inflammatory disease associated with psoriasis and high levels of comorbidity. There is no data on comorbidity in PsA with and without enthesitis.

Objectives: Evaluation of the frequency and the structure of comorbidity in PsA patients with and without enthesitis.

Methods: A retrospective analysis of 239 case histories of PsA patients observed at the Moscow City Rheumatology Center was performed. There were 68 (28.5\%) PsA patients with enthesitis and 171 (71.5\%) patients without enthesitis. The study included 132 female and 107 male patients, mean age was $52.0 \pm 14.8$ years. 217 patients $(90.8 \%)$ had psoriasis. The average psoriasis duration was $19 \pm 14.3$ years. The diagnosis of PsA was established based on CASPAR criteria (2006).

Results: Comorbid diseases were observed in 141 (59\%) patients with PsA. Moreover, in the group of PsA patients with enthesitis, comorbidity was noted much more often compared to the group without enthesitis $(70.6 \%$ and $54.4 \%$, respectively, $p=0.01$ ). The most common comorbid diseases were: arterial hypertension (25\%), gastropathy $(16.2 \%)$, osteoporosis $(13.2 \%)$, osteoarthritis $(11.8 \%)$, diabetes mellitus $(11.8 \%)$, hyperuricemia $(10,3 \%)$, obesity $(8.8 \%)$. A distinctive feature of PsA patients with enthesitis was a more frequent detection of osteoporosis $(13.2 \%$ and $5.8 \%$, respectively, $p=0.01$ ), which may be due to concomitant osteitis.

Conclusion: High rates of comorbidity were found in PsA patients with enthesitis $(70,6 \%)$. The most prevalent comorbid diseases were arterial hypertension $(25 \%)$, gastropathy $(16.2 \%)$ and osteoporosis (13.2\%). Patients with PsA with enthesitis were significantly more likely to have osteoporosis compared with patients without enthesitis, which is important to consider when managing patients.

Disclosure of Interests: None declared

DOI: 10.1136/annrheumdis-2020-eular.1396

\begin{tabular}{|l|l}
\hline AB0834 & CLINICAL CHARACTERISTICS OF \\
& PSORIATIC ARTHRITIS IN CHINESE PATIENTS: \\
& A CROSS-SECTIONAL OBSERVATIONAL \\
& STUDY
\end{tabular}

\section{Z. Song ${ }^{1}$, B. Li ${ }^{1}$, Z. Zhang ${ }^{1} .{ }^{1}$ Peking University First Hospital, Beijing, China}

Background: The clnical features of psoriatic arthritis(PsA)greatly varied in reports fomr different countries. There was no exact data in China.

Objectives: To disclose the characteristics of PsA in China, we initiated an investigation it in our cohort of PsA patients.

Methods: A cross-sectional observational study was conducted in our PsA cohort of Peking University First Hospital. All the clinical and imaging data at the patient's first visit were collected, including the age, gender, disease course, 